\title{
RNA interference in marine and freshwater sponges: actin knockdown in Tethya wilhelma and Ephydatia muelleri by ingested dsRNA expressing bacteria
}

\author{
Ajna S Rivera ${ }^{1,5+}$, Jörg U Hammel ${ }^{2 \dagger}$, Karri M Haen ${ }^{3}$, Elizabeth S Danka ${ }^{1,6}$, Brandon Cieniewicz $^{1,7}$, lan P Winters ${ }^{1}$, \\ Dora Posfai ${ }^{1}$, Gert Wörheide ${ }^{4}$, Dennis V Lavrov ${ }^{3}$, Scott W Knight ${ }^{1,8}$, Malcolm S Hill ${ }^{1}$, April L Hill ${ }^{1 *}$ and Michael Nickel ${ }^{2 *}$
}

\begin{abstract}
Background: The marine sponge Tethya wilhelma and the freshwater sponge Ephydatia muelleri are emerging model organisms to study evolution, gene regulation, development, and physiology in non-bilaterian animal systems. Thus far, functional methods (i.e., loss or gain of function) for these organisms have not been available.

Results: We show that soaking developing freshwater sponges in double-stranded RNA and/or feeding marine and freshwater sponges bacteria expressing double-stranded RNA can lead to RNA interference and reduction of targeted transcript levels. These methods, first utilized in C. elegans, have been adapted for the development and feeding style of easily cultured marine and freshwater poriferans. We demonstrate phenotypic changes result from 'knocking down' expression of the actin gene.
\end{abstract}

Conclusion: This technique provides an easy, efficient loss-of-function manipulation for developmental and gene regulatory studies in these important non-bilaterian animals.

\section{Background}

The ability to reconstruct the evolution of animals relies heavily on the availability of comparative data to facilitate understanding of evolutionary trends from the molecular to the organismal level. Among the non-bilaterian animals, recent utilization of phylogenomics has increased our understanding of metazoan relationships [1-5]. Comparative genomic approaches were used to reconstruct ancestral genomes like the putative genome of the last common ancestor of the Metazoa (LCAM) [6-8]. Moreover, the same genomic data is used to evaluate hypotheses regarding the evolution of metazoan-specific pathways like those involved in development [9-11] or cell signaling [12-15]. For example, the genome of the demosponge Amphimedon queenslandica is playing a key

\footnotetext{
* Correspondence: ahill2@richmond.edu; m.nickel@uni-jena.de

+ Contributed equally

'Department of Biology, University of Richmond, Richmond, VA, USA ${ }^{2}$ Institut für Spezielle Zoologie und Evolutionsbiologie mit Phyletischem Museum, Friedrich-Schiller-Universität, Jena, Germany

Full list of author information is available at the end of the article
}

role in deciphering evolutionary trends in Metazoan specific gene networks and pathways [e.g. $[8,16]]$. Nevertheless, evolutionary biology relies on more than genomic data. Functional studies based on reverse genetics techniques are needed to test the hypotheses provided by genomic analyses. However, the availability of genomic resources and reverse genetic techniques in the nonbilaterian animals is limited compared to the other animals (Figure 1, tree after [2,17]). For the Cnidaria, the Placozoa, and the Ctenophora genomic data and reverse genetic techniques are available [e.g. [18-22]]. However, thus far, only some genomic data is available for the Porifera, and the sister group of Metazoa, the Choanoflagellata. Reverse genetic techniques for these animal groups is of high priority for a comprehensive analysis of evolution and development of the non-bilaterian Metazoa.

Apart from permanent knockout and/or transgenic lines [e.g. [23]], RNA interference (RNAi) is a powerful tool to alter the expression of genes [24-26]. RNAi can be mediated in a variety of ways, for example soaking animals in double-stranded RNA (dsRNA) or morpholinos

\section{Biomed Central}




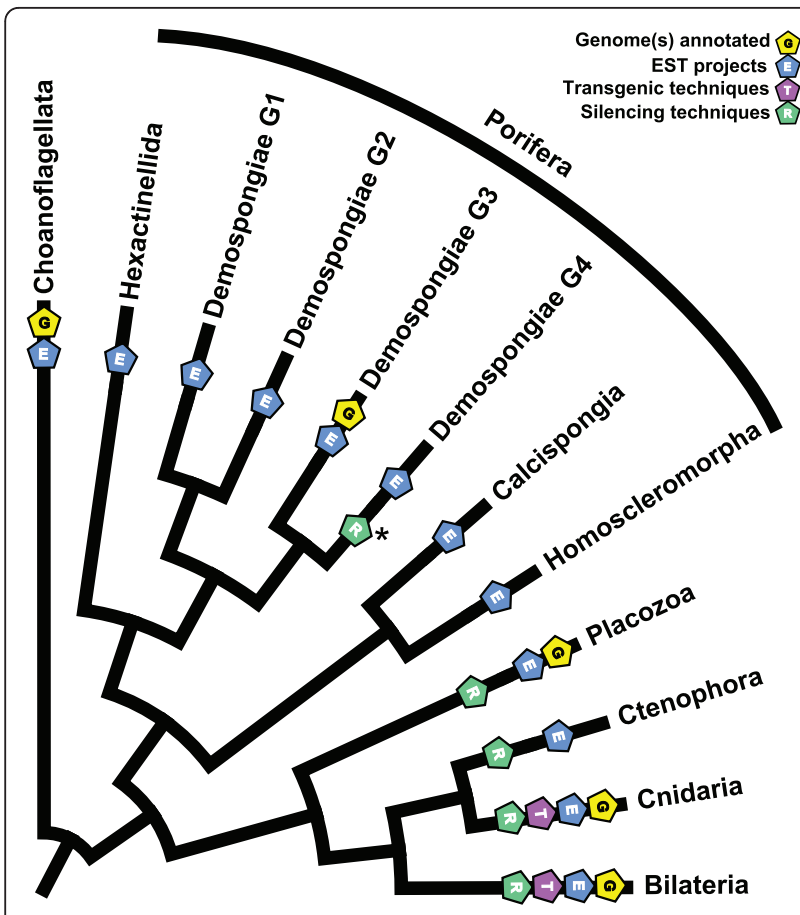

Figure 1 Genomic resources and reverse genetics methods presently available for representative model species of nonbilaterian Metazoa. Available methods for each group plotted on a cladogram (tree after $[2,17])$. While EST and/or genomic data are available for many representatives of each clade, functional (transgenic and silencing) techniques have only been adapted to a few non-bilaterian groups. The present study provides the first RNAi silencing protocols for a part of the Porifera, i.e. the two cultivatable Demospongiae G4 species Ephydatia muelleri and Tethya wilhelma (asterisk). There are no species currently maintainable in laboratory cultures among the demosponge clades $\mathrm{G} 1$ to $\mathrm{G} 3$, which would be an important prerequisite for future extensive RNAi studies.

$[18,19,27]$, injecting dsRNA or morpholinos [28,29], or feeding dsRNA expressing bacteria [24,25,30]. Among these techniques, certain limitations have to be considered: (1) Soaking animals in dsRNA appears to be most problematic in seawater due to issues of RNA stability. This might be one of the reasons why functional genomic studies in marine non-Bilateria are scarce. So far, soaking techniques have only been adapted to Eleutheria dichotoma, Aiptasia pallida and Trichoplax adhaerens $[18,19,27]$. In some studies, the salinity has been altered $[18,19]$, which cannot be tolerated by most marine organisms. (2) The injection of dsRNA or morpholinos is only efficient if early embryos can be investigated. (3) Feeding of dsRNA-expressing bacteria requires efficient uptake of bacteria either naturally or by mimicking other food sources [30].

Because sponges represent a key group for our understanding of Metazoan evolution including the changes in gene regulation that took place leading to multicellularity and complex body plans in this lineage, our aim was to test and establish RNAi protocols in one freshwater and one marine sponge species. By addressing both living modes we intended to provide a broad basis for future adaptations of this method to other sponge species.

The marine species Tethya wilhelma Sarà, Sarà, Nickel \& Brümmer, 2001 (Demospongiae, Hadromerida, Tethyidae) and the freshwater species Ephydatia muelleri (Lieberkühn, 1856) (Demospongiae, Haplosclerida, Spongillidae) are emerging basal animal model organisms. Ephydatia is an abundant and common sponge found on all major land masses in the world [31]. Recent research has lead to a better understanding of Ephydatia biology including cellular physiology and function as well as the role of conserved signaling pathways [e.g., [32-35]]. One of the most important aspects is that during winter Ephydatia forms thousands of gemmules which are cysts containing totipotent stem cells (archeocytes). These gemmules can be collected, stored at $4^{\circ} \mathrm{C}$ for up to one year, and easily induced to hatch into fully differentiated adults on the laboratory bench. This cultivation routine provides a system to study stem cell differentiation and animal body plan development in these animals [32,36]. A genome and a transcriptome are currently in the process of being sequenced for this species. Tethya wilhelma is one of the few marine sponges that can currently be permanently maintained in the laboratory based on its capability to reproduce asexually through buds [37-39]. In addition, this species has been studied on a broad scale including functional anatomy [40-42], contractile and movement behavior $[13,37,43]$, physiology of integration $[13,44,45]$ and bud development [39]. A transcriptome has been sequenced recently and is currently being annotated, and sequencing of the genome is in the planning stage.

To enable functional studies in sponges we developed a protocol for bacterial mediated RNAi-induced silencing based on gene silencing techniques developed for Caenorhabditis elegans [24,25]. The protocol has been adapted to the marine and freshwater environments and tested by knocking down cytoplasmic actin mRNA levels in juvenile specimens of T. wilhelma and E. muelleri. Our results indicate that this pathway is able to function in both marine and freshwater sponge species.

\section{Results and Discussion}

Actin sequences used for knockdown experiments

From a Tethya cDNA library, we identified a 707 bp fragment of $T$. wilhelma as a putative beta-actin ortho$\log (T w-a c t b ;$ Additional File 1, Figure S1). The Tw-actb fragment contains a putative 5'UTR (nt 1-126) and the $5^{\prime}$ part of the putative coding sequence (CDS) (nt 127$705)$. This sequence is quite similar to the Homo sapiens $\beta$-actin sequence (NM_001101 [46]) with 73.4\% pairwise identities overall. A motif search in the translated 
putative CDS region of $T w$-actb revealed PROSITE [47] Actin signature 1 (PS0046) and 3 (PS01132) at residues 54-64 and 105-117 respectively. Actin signature 2 (PS00432) has not been identified; it may reside in the uncloned 3' terminus. Using beta-actin degenerate PCR primers, we isolated a $338 \mathrm{bp}$ fragment of E. muelleri as a putative beta-actin ortholog (Em-actb; Additional File 1, Figure S1). The Em-actb fragment is $100 \%$ identical to the putative Tethya beta-actin ortholog at the amino acid level. Our Em-actb fragment also contains PROSITE actin signature 3 . Both fragments were cloned into the L4440 plasmid (Additional File 1, Figure S2) and transformed into Escherichia coli HT115(DE3), which was induced to produce dsRNA.

\section{In silico evaluation of the actin sequences used for knockdown experiments}

In order to ensure that we are targeting cytoplasmic actin and not another actin isoform or actin-related protein, we performed an in silico analysis. Using species specific BLAST searches [48] we translated both E. muelleri and T. wilhelma actin coding sequences and aligned them to other actins. These actin sequences were obtained from a protein sequence alignment used in a previous study [49] as well as contig translations (see Additional File 1, Figure S1, Additional File 1, Figure S2) based on tblastn searches using $H$. sapiens $\beta$-actin (AAH16045) against the following transcriptomes housed at NCBI dbEST [50]: Monosiga ovata (Choanoflagellata), Amphimedon queenslandica (Porifera, Demospongiae), Leucetta chagosensis (Porifera, Calcarea), Oscarella carmella (Porifera, Homoscleromorpha), Nematostella vectensis (Cnidaria, Anthozoa), Hydra vulgaris (Cnidaria, Hydrozoa), Mnemiopsis leidyi (Ctenophora), and Trichoplax adhaerens (Placozoa). The RaxML tree we generated from these data showed that both T. wilhelma and E. muelleri sequences clearly group within all other metazoan putative cytoplasmic Actins and are clearly separated from other Actins or Actin-related proteins (Additional File 1, Figure S3). An alignment of the partial coding sequences of $T w$-actb and Em-actb used for RNAi with 9 additional holozoan orthologs produced pairwise identity of $82.4 \%$ on the nucleotide level (Additional File 1, Figure S4) and $97.2 \%$ on the amino acid level (Additional File 1, Figure S5). RaxML tree and alignment analyses confirm that cytoplasmic actin was targeted in our RNAi knockdown experiments and thus we expect cytoplasmic actin specific phenotypic effects.

\section{Evaluation of a soaking method for dsRNA delivery}

We first attempted a soaking method for dsRNA delivery in E. muelleri. As negative controls, we used dsRNA for green fluorescent protein (GFP) since sponges do not express GFP. We also used double-stranded DNA (dsDNA) generated by PCR amplifying the E. muelleri beta-actin gene since dsDNA should not be processed through the RNAi pathway.

Soaking sponges in high concentrations $(>50 \mu \mathrm{g} / \mathrm{mL})$ of any nucleic acid (GFP dsRNA, actin dsRNA, actin dsDNA) leads to potentially cytotoxic effects to the sponges. Freshwater sponges are sensitive to changes in osmolarity [51], which could be the cause of the observed cytotoxicity. When exposed to high nucleic acid concentrations, the tissues regressed, choanocyte chambers seemed to collapse, and sponges ceased pumping. Thus, in titration experiments we tested for concentrations at which dsDNA and GFP dsRNA would not have an observable effect on sponge morphology, while actin dsRNA would. A concentration of $1-10 \mu \mathrm{g} / \mathrm{mL}$ of beta-actin dsRNA led to distinct observable morphologies; including loss of contact of the growing edge of tissue with the culture plate, loss of definition in the canal structure of the sponge, and poorly defined choanocyte chambers (Additional File 1, Figure S6A,B,C). Longer exposures to betaactin dsRNA resulted in more pronounced phenotypes (Additional File 1, Figure S6D,E,F). Control experiments with exposure to GFP dsRNA or beta-actin dsDNA at similar concentrations, did not exhibit phenotype changes (data not shown). Interestingly, removal of the dsRNA beta-actin solution and replacement with normal culture media lead to full recovery of the sponge tissue after one to two days including canals, distinct connections to the culture plate and a developed osculum (Additional File 1, Figure S6G,H,I,J).

Our data indicate that a soaking method is viable for induction of the RNAi pathway in E. muelleri. However, the tight range in the amount of dsRNA that seemed to be effective to produce an expected phenotype will require titration experiments for each gene addressed in future RNAi studies. We did not attempt a soaking method for T. wilhelma since it is necessary to alter the salinity (by a decrease of $\sim 50 \%$ ) of the culturing media in order to ensure RNA stability. We know that T. wilhelma and most other marine sponges do not tolerate salinity changes in this range without experiencing changes in contractile behavior and most likely phenotypic changes (JH \& MN, unpublished observations). Thus, a soaking method would likely not be viable for marine species.

\section{Feeding method for the freshwater sponge Ephydatia muelleri}

Since sponges naturally feed on bacteria, a feeding method for dsRNA delivery might be more universally applicable in marine and freshwater sponge systems. In E. muelleri, we fed sponges HT115(DE3)/L4440 E. coli expressing Em-actin dsRNA, for 24 hours. Afterwards, sponges were washed several times in culture media to remove bacteria and tissue was harvested within one to two hours (Figure 2A). Sponges were also fed HT115 

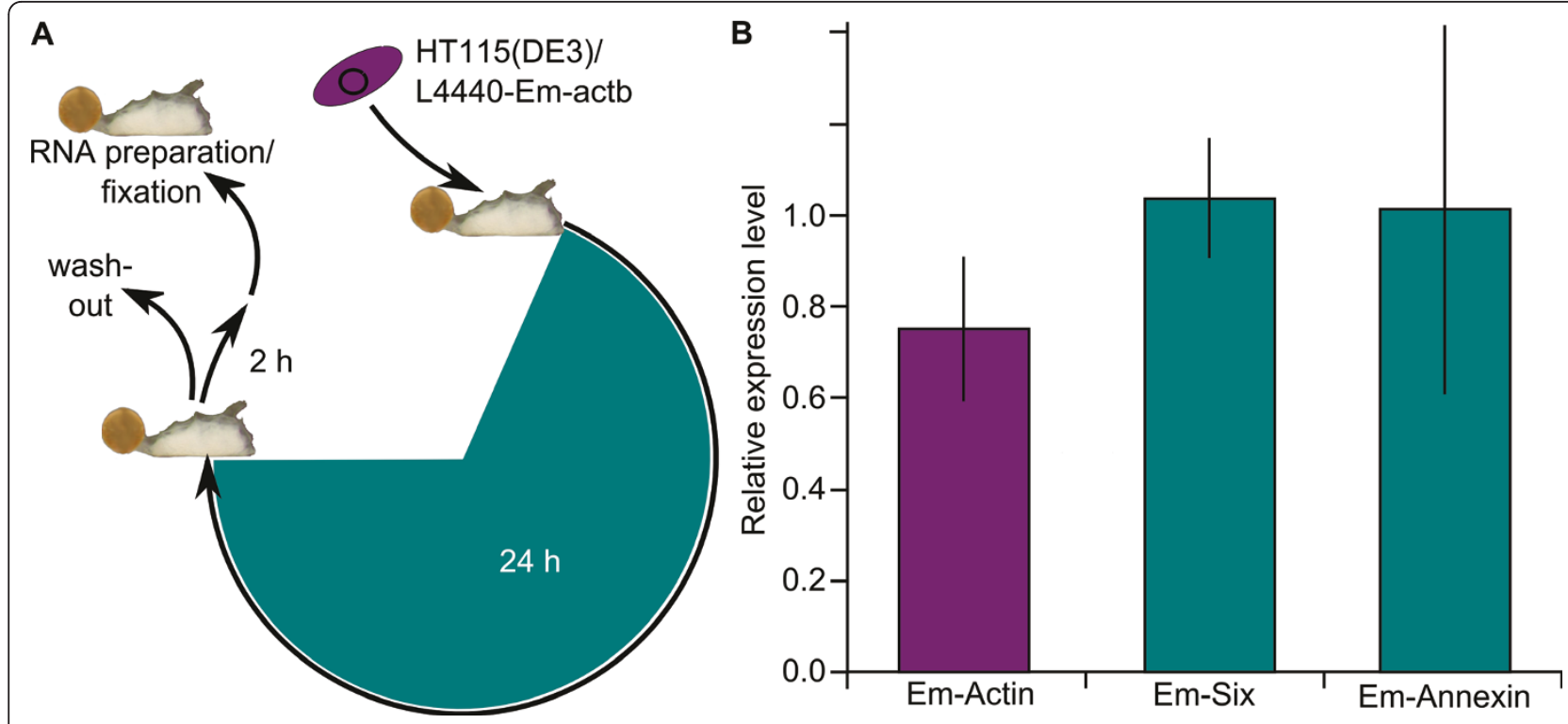

Figure 2 Experimental feeding scheme and quantitation of actin expression levels after RNAi treatment in Ephydatia muelleri. A. After feeding for 24 hours with induced HT115(DE3)/L4440-Em-actb or non-induced controls, sponges were washed several times in 1X Strekal's medium, photographed, and harvested for RNA preparation or fixation within 1-2 hour. B. qRT-PCR assay for gene expression levels of Em-actin (purple bar) and two control genes. (Em-six and Em-annexin, green bars) after dsRNA treatment with induced HT115(DE3)/L4440-Em-actb. Levels were normalized to Ef1 $\alpha$ and gene expression levels are given as relative levels of RNAi treated sponges against control sponges. Combined data with $N_{E m-a c t i n}=11, N_{E m-S i x}=3, N_{E m-A n n e x i n}=3$; bars represent standard deviations.

(DE3) or bacteria expressing GFP to monitor feeding behavior and to test for a response to induced bacteria. GFP-fed sponges did not show any response that differed from sponges fed bacteria lacking expression vector (data not shown).

To test the ability of actin dsRNA to reduce actin expression, we performed quantitative-RT-PCR (qRTPCR) assays. For qRT-PCR analysis, we fed sponges either dsRNA-expressing bacteria, untransformed bacteria, or GFP-expressing bacteria for 24 hours. Sponge tissue was harvested and we utilzed qRT-PCR to access actin expression levels. A relative quantification procedure was used to calculate a ratio of treatment to control expression levels, either for the gene of interest (i.e., Em-actb) or for the non-target genes (i.e., Em-six and Em-annexin). If the target gene was not affected by actin RNAi we would expect a ratio of 1 . The ratio of paired treatment and control expression levels were averaged and are shown in Figure 2B. We performed eleven total qRT-PCR experiments with L4440-Em-actb (abbreviated $d s E m$ - $a c t b$ )-fed sponges and controls. In ten of these experiments, Em-actb levels were lower in dsRNA-fed sponges than controls, when normalized to the housekeeping gene EF1-alpha expression levels. These results were significant $(\mathrm{p}<0.05)$ as the relative Em-actb mRNA expression levels dropped down to $75 \%$ of the control values (thus, average knockdown in RNAi-fed sponges was near $25 \%$, with a maximum knockdown value of $\sim 55 \%$ ). Longer treatments (48 hours) with the same bacteria did not lead to a greater effect (data not shown).

To test for nonspecific effects, we repeated the qRT-PCR experiment using sponges fed bacteria expressing dsEm$a c t b$ and then assayed expression levels for Em-annexin and Em-six1/2. Em-Annexin is a structural protein expressed in the archaeocytes, and in developing and mature choanocyte chambers [36] while Em-Six is a transcription factor expressed in multiple cell types including archaeocytes and choanocytes throughout development (AH, unpublished data). Of three separate experiments totaling about 15 sponges per condition, Em-annexin and Em-six mRNA levels were not significantly different in $d s E m$-actb-treated sponges compared to HT115(DE3)-fed control sponges (Figure 2B). Any differences were small and non-directional, suggesting that dsEm-actb did not affect Em-annexin or Em-six expression.

To further demonstrate, on the molecular level, that it is possible to knockdown genes other than actin, we present RT-PCR, qRT-PCR and Western blot data for experiments conducted where sponges were either fed bacteria or soaked with dsRNA directed to Em-annexin and Em-six genes. We used RT-PCR and gel-based analysis to evaluate dsEm-annexin-treated sponges using both the soaking (sponges soaked in $10 \mu \mathrm{g} / \mathrm{mL}$ Em-annexin dsRNA) and feeding-based methods (described above). In both cases, reduced expression of Em-annexin is apparent when compared with control-treated sponges (Additional File 1, 
Figure S7A). We used qRT-PCR to demonstrate that sponges fed HT115(DE3)/L4440 Em-six dsRNA constructs showed reduced Em-six expression when compared to control-treated tissues. These results were significant and the relative Em-Six mRNA expression levels dropped down to $\sim 69 \%$ of the control values (thus, average knockdown in RNAi-fed sponges was $\sim 31 \%$; with a maximum knockdown value of $\sim 70 \%$ ) (Additional File 1, Figure S7B). It is clear that several parameters will need to be optimized to consistently achieve $>50 \%$ knockdown. These include time and length of exposure to dsRNA, age of sponges and ability to consistently induce bacteria and control for amount of bacteria fed to sponges.

To demonstrate that knockdown is also evident at the protein level, we performed Western blot analysis for both Actin and Six proteins after treatment with either $d s E m-a c t b$ or $d s E m$-six RNAi (Additional File 1, Figure S8). The variation in knockdown levels for Actin protein found in Western analysis was consistent with the variation in gene expression levels found by qRT-PCR after $d s E m-a c t b$ treatment. Two different representative Western blots showing $d s E m$-actb-treated tissue compared to control-treated tissue are shown and illustrate that Actin protein levels are lower after $d s E m$ - $a c t b$ treatment (Additional File 1, Figure S8A). In response to $d s E m$-six treatment, protein levels for Six were also reduced while the same blot showed that Actin protein levels remain relatively constant (Additional File 1, Figure S8B).

\section{Feeding method for the marine sponge Tethya wilhelma}

Using the same bacteria and a similar expression vector construct as described above, but a different feeding profile (Figure 3A) we fed T. wilhelma specimens $T w$-actb dsRNA expressing HT115(DE3). Larger specimens of $T$. wilhelma were cultured over a period of 33 and 57 hours, respectively, and pulse-fed with ds $T w$ - $a c t b$ expressing bacteria or a control strain for 4.5 hours each day. This resulted in actin knockdown in RNAi-treated sponges (Figure 3B). After 33 hours of cultivation, including two pulse-feeding cycles, relative $T w$-actb expression levels dropped down to $67.5 \%$ in relation to control animals, representing an average knockdown by $32.5 \%$. After an additional dsRNA feeding pulse within a total cultivation time of 57 hours, the relative actin mRNA levels remained within a reduced range of $76.9 \%$ in relation to control animals. Thus, all ds Tw-actb-treated specimens $(\mathrm{N}=6)$ showed significant reductions compared to endogenous mRNA levels (dependent t-test, $\mathrm{p}<0.05$ ). In contrast, pulse feeding of dsTw-actb expressing bacteria for a reduced time of 20 minutes did not reveal any knockdown effect within 33 or 57 hours (data not shown) for this species.

To determine if greater knockdown is observed in T. wilhelma after longer-term exposure to $d s T w$-actb, we repeated the experiment over a period of eight days. Between 81 and 201 hours after initial feeding, single specimens were tested for relative $T w$-act $b$ expression levels, which were between $87.6 \%$ (at $81 \mathrm{~h}$ ) to $50.4 \%$ (at $105 \mathrm{~h}$ ) to the level of $T w$ - $a c t b$ in control sponges. While this is a wide range, the average $(68.7 \% \pm 23 \%$; $\mathrm{N}=5)$, is close to the total average of the short-term experiment $(72.2 \% \pm 24 \% ; \mathrm{N}=6)$, which represents significant reductions in expression levels (dependent t-test, $\mathrm{p}<0.05)$.

To determine viability of sponges affected by longterm actin knockdown, all specimens that were used for quantification in the long-term experiment were timelapse imaged during the 24-hour period preceding sampling and preparation for RNA quantification. All specimens displayed typical contractile behavior (Additional File 1, Figure S9, Additional File 2) demonstrating the general viability of the RNAi-treated specimens. Thus, our results demonstrate that daily pulse feeding for 4.5 hours does not result in lethal effects as it has been observed by direct dsRNA incubation in a dose-dependent manner [27].

Evaluation of morphological changes in response to actin 'knockdown' in Ephydatia muelleri

To examine morphological changes due to actin knockdown in E. muelleri, we stained dsEm-actb-treated sponges and control sponges with Bodipy-558/568 conjugated phalloidin. We examined three major regions of the sponge tissue by confocal laser scanning microscopy (cLSM): 1) the outer edge of sponge growth, 2) the regions containing newly developed choanocyte chambers, and 3) the endopinacocyte-lined canals surrounded by choanocyte chambers. An extensive Actin network was present at the edge of sponge growth. The outer surface of the sponge that forms attachments to the substrate is composed of exopinacoderm and basopinacoderm and is made of continuous epithelium where some connective and migratory (amoebocytes) cells are observed. The region also contains filopodia of multipolar cells leading from the mesohyl to the exopinacoderm. Small foci represent the edge of two abutting cells when viewed from above, and larger foci likely represent focal adhesion points where Actin bundles highlight filopodia of mesohyl cells. Sponges fed dsEm- $a c t b$ have a less extensive Actin network at the growing edge of the sponge. Many fibers do not run continuously through the sponge tissue and the continuous fibers often lack the network organization seen in control sponges. These data likely indicate that there is a reduced cellular mobility in the mesohyl (Figure 4 A \& D).

The sponge region containing newly developed choanocyte chambers (choanosome) was chosen as it is a relatively thin region but allows us to see the overall pattern of developed sponge tissue. In this region the Actin-rich choanocytes themselves look very similar in 


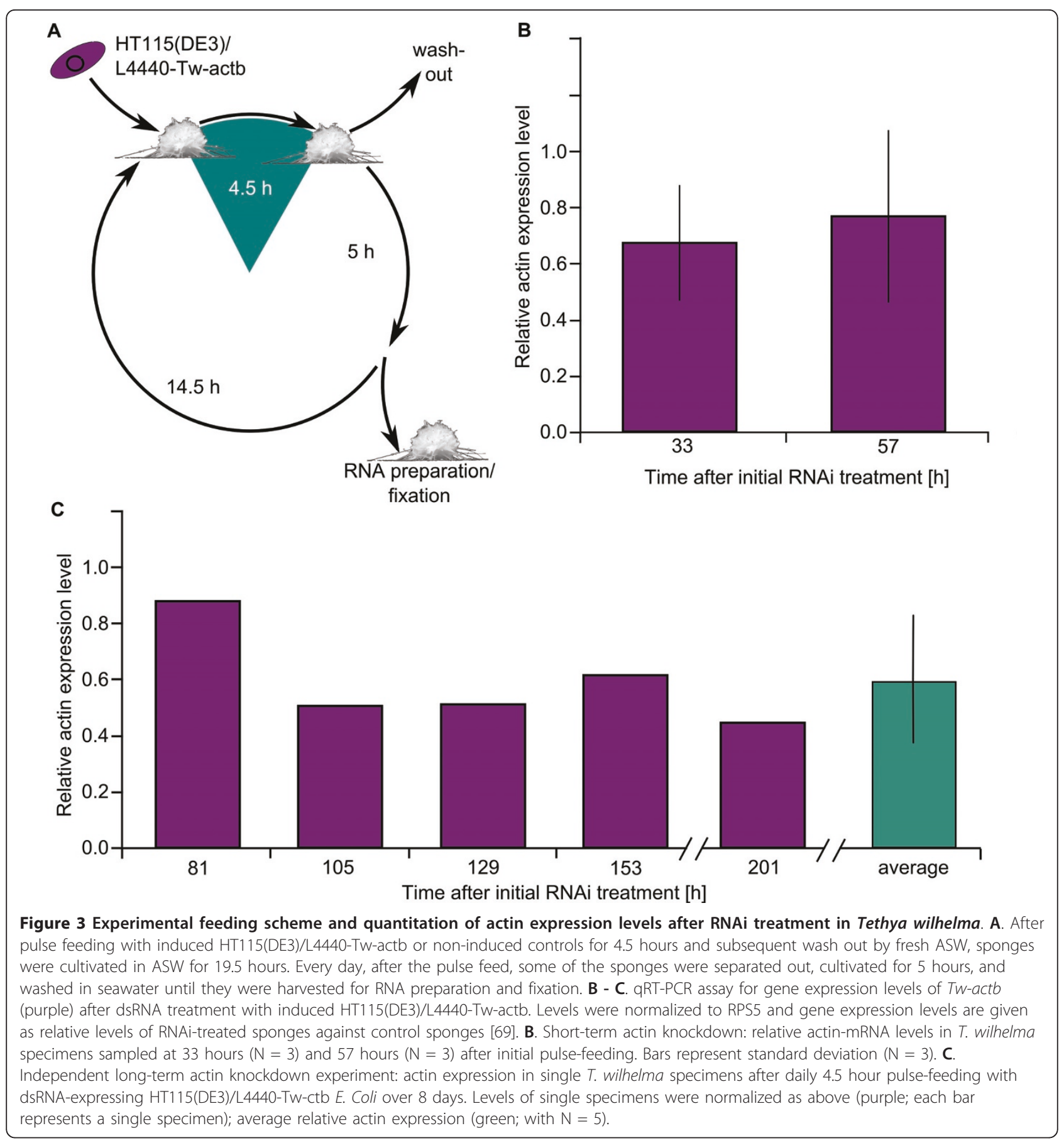

treated and control sponges. To further investigate, we performed transmission electron microscopy on a control and a $d s E m$-actb-treated sponge to see whether ultrastructural aspects of choanocytes were affected after RNAi treatment. While a more thorough sampling strategy is required, we found one trait in the microvillar collar that may differ between treatment and control sponges. Actin RNAi-treated choanocytes had a greater percentage of cells with misshapen/folded collars
(Additional File 1, Figure S10). The control sponges, on the other hand, contained numerous perfectly round collars, which were not observed in the Actin RNAitreated sponges (Additional File 1, Figure S10). However, the pattern of endopinacocytes connecting the choanocytes differed markedly. Control sponges have canals that are clearly lined by Actin-rich endopinacocytes. dsEm-actb-fed sponge canals are far less clearly lined (Figure $4 \mathrm{~B} \& \mathrm{E}$ ). In dsEm-actb-treated sponges, 

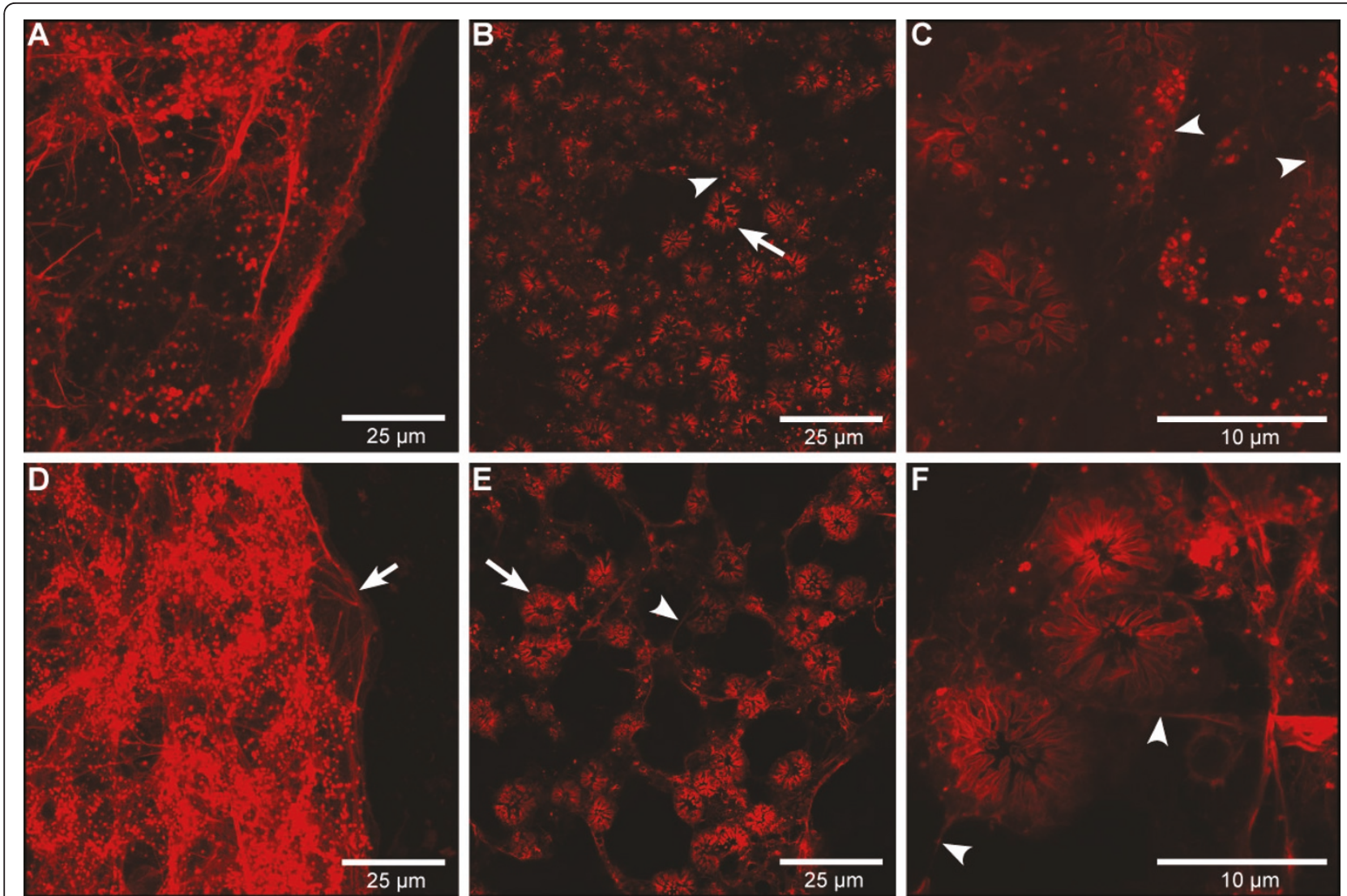

Figure 4 Phalloidin-stained tissue in control vs. actin RNAi-treated Ephydatia muelleri. Confocal laser scanning microscopy images of BODIPY 558/568 phalloidin conjugate-stained whole-mount preparations of E. muelleri. A - C. Actin RNAi-treated sponge tissues. D - F. Controltreated sponges. A and D. Arrow shows Actin filament connections to substrate. These connections are generally missing in RNAi-treated tissues. $\mathrm{B}$ and $\mathrm{E}$. Arrows show choanocyte chambers while arrowheads point to Actin connections (in endopinacocytes) between chambers, which are largely missing in treated sponges. C and F. Arrowheads show extensive Actin connections between and around choanocyte chambers. These connections are missing or not as extensive in treated tissues.

the endopinacocytes that span the spaces between choanocytes are thin and display a weaker Actin signal.

The Actin cytoskeletal network is thus reduced in dsEm$a c t b$-fed pinacocytes, but the choanocytes appear to be unaffected at the level observed with light microscopy. In the canals lined by Actin-containing pinacocytes, the Actin fibers run continuously around the canals, over the span of many cells. Control sponges have clearly outlined canals in most focal planes. dsEm-actb-fed sponges, however, have canals that are not as well-defined and are lacking clear phalloidin-stained pinacocytes in many planes (Figure 4 C\&F). Overall, our observations suggest a general knockdown of the Actin cytoskeleton with a less prominent Actin network.

\section{Evaluation of morphological changes in response to actin 'knockdown' in Tethya wilhelma}

To examine morphological changes due to actin knockdown in $T$. wilhelma, we fixed sponges that had been pulse-fed induced HT115(DE3)/Tw-actb bacteria expressing dsRNA and control HT115(DE3) bacteria daily
(Figure 3) after 33 and 57 hours. Using whole-mount preparations we examined two major regions of the sponge by cLSM that were stained using Alexa-488-conjugated phalloidin: 1) the exopinacocyte, and 2) the cortical mesohyl.

Phalloidin staining revealed an extensive Actin network in the exopinacocytes with Actin-rich ripple-like structures on the surface of the exopinacocytes (Figure 5A), structures typical in T. wilhelma and resembling lamellipodia extensions of fibroblasts (see Additional File 1, Figure S11). In the dsTw-actb-treated specimens, after 33 hours the exopinacocytes showed fewer Actin-rich ripples (Figure 5B) than in the control, while after 57 hours, ripples were almost absent (Figure $5 \mathrm{C}$ ). In addition, the Actin network was overall less prominent in the treated sponges than in the control specimens.

The largest effect was observed in the cortical mesohyl. The control sponges showed a large number of extremely long spindle-shaped bipolar cells (Figure 5D), which are typical morphotypes of highly mobile cells [52] 'frozen' in 
movement by fixation. In contrast, the ds $T w$-actb-treated sponges were characterized by atypical, extremely large atrophic multipolar cells (Figure 5E) and only few bipolar cells. In comparison, multipolar cells in the control sponges were much smaller (Figure 5F).

The changes in the pinacocytes with the reduced Actin-rich lammellae and atypical mesohyle with the atrophic multipolar cells and the lack of bipolar cells can be explained by decreased actin expression. While the Actin turnover rate is not high enough to produce lethal effects or cessastion of contractility, processes based on Actin dynamics like cell mobility or lamellipodia formation seem to be strongly affected. Since the atrophic multipolar cells correlate with a lack of mobile bipolar cells, it seemed that both forms actually represent extreme morphotypes of a single archeocyte-like cell type.

\section{Conclusions}

For the first time, we report successful RNAi techniques for gene knockdown in the sponges E. muelleri (freshwater) and T. wilhelma (marine). We used dsRNA soaking methodology for E. muelleri and a bacterial feeding method adapted to freshwater and marine culture media for both species. Our experiments demonstrate a significant reduction of endogenous mRNA levels in both species after feeding and represent the successful proof-ofprinciple for bacteria-mediated feeding induced RNA interference in marine and freshwater demosponges. We tested dsRNA methodology using cytoplasmic actin orthologs, a ubiquitously expressed gene involved in cell shape and motility.

In E. muelleri, qRT-PCR analysis showed a modest $(\sim 25 \%)$ but significant $(\mathrm{p}<0.05)$ knockdown of Em-actb after feeding sponges bacteria expressing dsRNA against Em-actb for 24 hours compared to controls, with a maximum knockdown of about 55\%. T. wilhelma sponges treated for 33 or 57 hours showed similarly modest but significant $(\mathrm{p}<0.05)$ reductions. T. wilhema treated for up to 8 days showed actin mRNA reductions throughout the entire period of the experiment. The maximum knockdown caused by dsTw-actb-expressing bacteria was almost 50\% compared to the control-group mRNA level. We also demonstrated in E. muelleri, at both the mRNA and protein level, that it is possible to knock down additional genes (Em-annexin and Em-six), thus illustrating this method should be applicable for other target genes.

The reduction in expression for actin demonstrates an efficient silencing for a highly expressed gene that may account for about $5 \%$ of the total protein content of a cell [53]. Furthermore, in order to increase the level of reduction, longer periods of treatment with dsRNA might be required for highly expressed genes or stable proteins like Actin. Hints of this were seen in the long- term T. wilhelma feeding and E. muelleri soaking experiments (Figure 3C, Figure S6, Additional File 1). It is possible that the RNAi effect may be greater than measured, because the knockdown effect may only be measured in surviving cells. Sponges are known to have high proliferation activity, a short cell cycle duration and cell shedding that is predominantly confined to the choanocytes [e.g. [54]]. This high cell turnover rate may be part of the reason that actin (or probably any gene) will not be knocked down at great levels. Due to the biology of these organisms and the method used, feeding sponges dsRNA will likely be a technique that can be utilized to knockdown, but not knockout gene expression. However, the overall significantly reduced mRNA levels during the entire period of the experiment clearly demonstrated the potential of bacterial-mediated RNAi-induced silencing for functional gene knock down studies in poriferans.

After feeding Em-actin dsRNA, E. muelleri did not display a macroscopic phenotype that could be clearly described at the cellular level. However, the primary Actin knockdown phenotype shown by phalloidin staining was a less extensive Actin network that was manifested in a "crumpled" edge, where tissues should be adhering to the substrate and migrating outwards. Additionally, a reduction of cellular connections in the choanocyte-rich regions and canals due to loss of Actin in endopinacocytes was observed. T. wilhelma showed neither a reduced survival rate nor alterations of rhythm or kinetics of body contractions. Like in E. muelleri, RNAi treated specimens of $T$. wilhelma displayed no distinct macroscopic phenotype. However, we found strong effects on mesohyle and pinacoderm cell anatomy of T. wilhelma. Apart from reduced Actin content and altered Actin network appearance in pinacocytes, the most prominent effects were hypertrophic mesohyle cells and the lack of mobile bipolar cells, indicating a reduced mobility and cell shape dynamics in dsRNAtreated specimens.

RNAi methods allow us to begin to study sponge gene function in a directed manner, including questions regarding evolution of gene regulatory networks, nervous system and muscle evolution, and also functional studies concerning biomineralization and other metabolic processes. Our results also suggest that RNAi might be a valuable tool to investigate cell-type functionality in sponges, as has been shown for other organisms, like Hydra [e.g. [30]]. This would facilitate studies on the evolution of cell differentiation in sponges. Additionally, it might help us to differentiate variable morphotypes of one single cell type versus other cell types. Thus, RNAi would help solve a long-standing problem for sponge biologists and evolutionary biologists interested in cell type evolution [e.g. [55]] by allowing unique studies to 

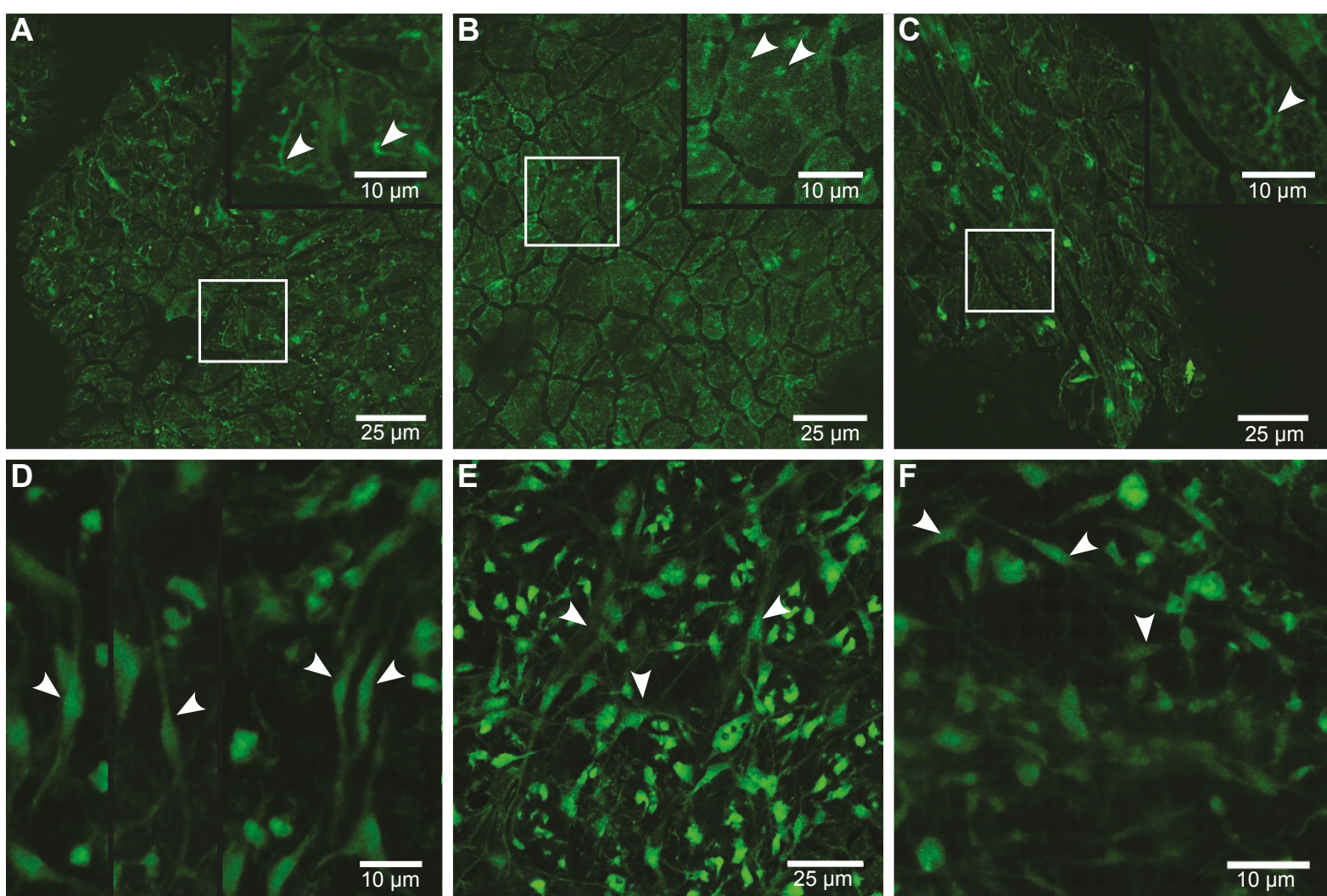

Figure 5 Phalloidin-stained tissue in control vs. actin RNAi-treated Tethya wilhelma. Confocal laser scanning microscopy images of Alexa 488 phalloidin conjugate-stained whole mount preparations of T. wilhelma. A, D and F. Control treated sponges. B and E. Actin RNAi treated sponges 33 hours after initial feeding (see feeding scheme in Figure 3). C. Actin RNAi treated sponge tissues 57 hours after initial feeding. A - C. Exopinacoderm layers showing pinacocytes. Insets display details of the main image. Arrowheads show Actin ripples at the cell surface as parts of the pinacocyte Actin network. Note: due to slight hypoosmotic fixative in order to avoid cell shrinking, the sponges are slightly inflated during fixation, forming small artifactual gaps between pinacocytes due to their extremely thin contact zones between the pinacocytes. This slight artifact is systemic in all samples and does not interfere with the characters important in the context (see Additional File 1, Figure S11 for more details). D. Three detail images of bipolar (moving) mesohyle cells (arrowheads) in control sponges. E - F. Multipolar cells (arrowheads): hypertrophic, large cells in RNAi-treated sponges (E) and normal multipolar cells in control sponges (F).

clearly define and distinguish sponge cell types, most likely in conjunction with in situ hybridization. We assume that our protocol will be readily applicable to other marine sponge species like the homoscleromorph Oscarella lobularis $[56,57]$ or the calcareous Sycon ciliatum and S. raphanus [58-60]. It will also be adaptable to the freshwater species E. fluviatilis and Spongilla lacustris, which are also commonly used to study sponge biology [36,61-63]. In conclusion, our present study closed an existing gap for a functional genomics protocol for the Porifera and will thus stimulate a number of comparative functional studies, which will deepen our understanding of metazoan evolution.

\section{Methods}

\section{Sponges}

E. muelleri gemmules were harvested from sponges collected from a dam outflow near Griswold, Connecticut, USA $\left(41^{\circ} 35^{\prime} 4^{\prime \prime} \mathrm{N}, 71^{\circ} 55^{\prime} 15^{\prime \prime} \mathrm{W}\right)$. Gemmules were washed in 3\% hydrogen peroxide, and re-washed several times in sterile, cold, 1X Strekal's [64] medium and stored at $4^{\circ} \mathrm{C}$ in the dark until use. Washed gemmules from the freshwater sponge E. muelleri are placed in 12-well plates containing $2 \mathrm{~mL}$ of $1 \mathrm{x}$ Strekal's medium and kept in the dark at $\sim 25^{\circ} \mathrm{C}$ for hatching. After hatching (2-3 days after plating) the $1 \mathrm{x}$ Strekal's was replaced daily. Gemmules were allowed to develop to stage 5 before they were treated with either dsRNA or fed bacteria to ensure that feeding structures had fully developed.

T. wilhelma originated from its type locality, the aquarium of the Zoologisch-Botanischer Garten Wilhelma Stuttgart (Germany) [38]. Due to its asexual reproduction by budding $T$. wilhelma was permanently cultured in the laboratory in Jena in tropical seawater aquaria under constant moderate flow conditions at a temperature of $24^{\circ} \mathrm{C}$ and a light regime of $12 \mathrm{~h}$ day and night. Artificial seawater (ASW; Tropic Marine, Wartenberg, Germany; dissolved in demineralized water) was partly renewed 
every few weeks. During cultivation, sponges were fed on a daily basis using finely dispersed commercial aquarium invertebrate food ('Artificial Plankton' Aquakultur Genzel, Freiberg am Neckar, Germany). For experiments, we used fully grown sponges ('adults') and stage-4 buds around $1 \mathrm{~mm}$ diameter, which were released shortly before the experiment, as well as juvenile sponges of up to $3 \mathrm{~mm}$ [for details on bud developments see ref. [39]].

\section{EST library construction and sequencing}

Several specimens of $T$. wilhelma were sampled from the aquarium of the Zoologisch-Botanischer Garten Wilhelma Stuttgart (Germany) and immediately frozen in liquid nitrogen. They were stored at $-80^{\circ} \mathrm{C}$ and shipped on dry ice to Iowa State University (Ames, Iowa, USA). Immediately after arrival, the samples were stored at $-80^{\circ} \mathrm{C}$ until they could be re-immersed in liquid nitrogen and ground with an RNase-free mortar and pestle. The following protocols for RNA isolation and cDNA synthesis used reagents from Invitrogen (Carlsbad, CA, USA): $1 \mathrm{~mL}$ of TRIzol Reagent was added for every $50 \mathrm{mg}$ of ground tissue, and total RNA extraction was carried out according to the manufacturer's instructions. Resultant RNA pellets were dissolved in $25 \mu \mathrm{L}$ of DEPC-treated water, with the addition of $1 \mu \mathrm{L}$ RNasin RNA protectant, and checked for RNA integrity on an agarose gel. mRNA was isolated from total RNA aliquots using the FastTrack MAG mRNA Isolation Kit with a Magna-Sep magnetic particle separator. mRNAs were treated with Calf Intestinal Phosphatase (CIP) to dephosphorylate uncapped 5' ends and processed with the GeneRacer kit for firststrand synthesis of full-length cDNAs using SuperScript III reverse transcriptase (RT). Second-strand synthesis was carried out using standard kit primers and Platinum Taq HiFi DNA polymerase. Size fractionation of cDNAs was achieved by gel-extracting cDNA pools of successive sizes with the PureLink kit. cDNA pools were then treated for blunt-end ligation and cloned with the Zero Blunt TOPO PCR Cloning Kit for sequencing. Random clone libraries were grown overnight in 96-well culture plates and submitted to the Iowa State University DNA Sequencing Facility for automated plasmid extraction and sequencing of cDNA inserts. Resulting sequence data was queried against the NCBI GenBank for identification of cDNA sequences with known function.

\section{Maximum likelihood gene tree analysis}

Using the cloned Tw-actb and Em-actb sequences, blastn searches were performed in a recently constructed EST database (T. wilhelma) and in Genbank (E. muelleri, organism-specific search). The dataset was complemented by organism-specific tblastn searches using $H$. sapiens $\beta$-actin (AAH16045). Contigs were created using Geneious Pro 4.74 (Biomatters, Auckland, NZ) using the assembly function; assemblies were visually inspected and corrected manually for errors caused by the automatic assembly. The contig sequence was analyzed for the actin open reading frame (ORF) starting with the amino acid sequence MDDXVAALV. The translated ORF was added to a previously published actin and actin-related protein amino acid sequence alignment [49]. Sequences were submitted to Genbank (T. wilhelma: HQ693078, E. muelleri HQ677599).

Additional cytoplasmic actin contigs and amino acid translations (see Additional File 3, Additional File 4) were created in the same way by organism-specific tblastn searches using $H$. sapiens $\beta$-actin (AAH16045) for the following early-branching Metazoa or Holozoa, included in the alignment: Amphimedon queenslandica; Hydra vulgaris; Leucetta chagosensis; Mnemiopsis leidyi; Monosiga ovata; Nematostella vectensis; Oscarella carmela; Oopsacas minuta; Trichoplax adhaerens.

The existing amino acid alignment was expanded using the Muscle algorithm of Geneious Pro. A rapid bootstrap analysis [65] on 100 replicates followed by search for the maximum likelihood tree was performed using RAxML 7.2.6 (PTHREADS) with the GTR (General Time Reversible) amino acid substitution model with site-specific evolutionary rates (4 discrete GAMMA categories). RAxML was called as follows: ./raxmlHPC-PTHREADS-SSE3 -T 4 -f a -x 12345 -p 12345 -\# 100 -m PROTGAMMAGTR -o At-arp4. The unrooted gene tree was visualized using Geneious Pro.

\section{RNAi constructs}

PCR primers for producing dsRNA for E. muelleri betaactin to use with a soaking method were designed with T3 or T7 RNA polymerase sequences on either end. Primer sequence for the forward primer included a T3 site (AATTAACCCTCACTAAAGG) on the 5 ' end followed by a beta-actin sequence of 5'-GCCCCCGAGGAGCACCCAGTCTT-3' and for the reverse primer a T7 site (TAATACGACTCACTATAGGG) with a beta-actin primer sequence of 5'-TCGGCAGTGGTGGTGAAAGAGTA-3'. PCR amplification yielded a 326-bp band, which was concentrated with a sodium acetate and ethanol precipitation. The PCR product was used as a template in RNA transcription reactions using $\mathrm{T} 3$ and $\mathrm{T} 7$ polymerase. The resulting single-stranded RNA transcripts were purified by phenol:chloroform extraction and quantified (Nanodrop). Equal amounts of T3 and T7 reactions were annealed to create double-stranded fragments by boiling for 3 minutes and then cooling slowly. dsRNA was precipitated and resuspended in 1X Strekal's before use, and dsRNA was confirmed on a $5 \%$ PAGE gel.

To produce constructs for bacterial feeding-mediated RNA interference in E. muelleri and T. wilhelma we used a protocol available from the Fire lab (http://firelab.stanford. edu/links.html) [24]. For E. muelleri a 290-nt product of the Ephydatia beta-actin gene, Em-actb (forward primer: 
5'-GGCCCCCACTCAACCCAAAGG-3'; reverse primer: 5'CCACGCTCGGTCAGGATCTT3') was placed in the L4440 Plasmid (FireLab Vector Kit, Addgene Plasmid 1654). The L4440 (pPD129.36) vector contains two convergent T7 polymerase promoters in opposite orientation separated by a multiple cloning site [25]. In order to grow cells for expression of dsRNA and feeding experiments the expression vector was transformed into HT115(DE3) E. coli (which is unable to degrade dsRNA). This line was named L4440-Em-actb (abbreviated here as dsEm-actb). For bacterial feeding-mediated RNA interference in $T$. wilhelma a 703-bp fragment of T. wilhelma actin gene was placed in the L4440 plasmid. As a control, HB101 E. coli transformed with the pLR71 GFP constructs were fed to E. muelleri (results not shown). T. wilhelma were fed noninduced HT115(DE3)/L4440-actb E. coli cells as a control. Primers and constructs used for control experiments with Em-annexin and Em-six are available on request.

\section{RNAi-induced silencing}

E. muelleri sponges treated with dsRNA for the soaking method were used 5-6 days after plating when the sponges have fully developed oscula (indicating that they will efficiently pump water through their tissues and feed on ingested bacteria). Strekal's culture medium was removed and replaced with dsRNA resuspended in $1 \mathrm{X}$ Strekal's and diluted appropriately. A range of dsRNA concentrations were used as described in the results section.

For feeding E. muelleri bacteria expressing dsRNA, $5 \mathrm{~mL}$ overnight cultures in LB broth were inoculated with L440$\mathrm{Em}$-actb one day prior to the sponge feeding stage. The next day, each overnight culture was inoculated in $100 \mathrm{~mL}$ $\mathrm{LB}$ broth with ampicillin and maintained at $37^{\circ} \mathrm{C}$ with shaking. Bacterial cultures were induced with $19 \mathrm{mg} / 100 \mathrm{~mL}$ of Isopropyl- $\beta$-D-thiogalactopyranoside (IPTG) at $\mathrm{OD}_{595} 0.4$ $(\sim 3 \mathrm{hrs}$ after scale up) to begin bacterial production of dsRNA. Cells were induced for 1.5 hours. $50 \mathrm{~mL}$ of cells from each overnight culture were centrifuged in conical tubes for 8 minutes at $4000 \mathrm{rpm}$ at $4^{\circ} \mathrm{C}$. The supernatant was discarded and the bacterial pellet was resuspended in $50 \mathrm{~mL} \mathrm{1x}$ Strekal's media. This constituted the feeding stock. From this stock dilutions were made for each treatment to a concentration of $\sim 1 \times 10^{8}$ cells $/ \mathrm{mL}$. The $1 \mathrm{x}$ Strekal's culture medium was removed from each sponge and 1 $\mathrm{mL}$ of the appropriate dilution of bacteria producing dsRNA was added. Sponges were cultured for 24 hours with bacteria producing dsRNA. After 24 hours, sponges were washed several times in $1 \mathrm{X}$ Strekal's medium, photographed on a stereomicroscope and then harvested for RNA after 1-2 hours.

For dsRNA-feeding experiments in T. wilhelma, a $10 \mathrm{ml}$ $2 \mathrm{xYT}$ culture containing appropriate antibiotics was inoculated with a single colony of HT115(DE3)/L4440actb and cultured overnight at $37^{\circ} \mathrm{C}$. The culture was diluted 160-fold and allowed to grow to $\mathrm{OD}_{600}=0.4$ before IPTG was added to a final concentration of $0.4 \mathrm{mM}$ in order to induce actin dsRNA expression. Incubation of the culture continued for additional 5 hours at $37^{\circ} \mathrm{C}$. Cells were harvested by centrifugation and transferred to ASW immediately prior to application on T. wilhelma. Stage-4 buds [39] and larger specimens of T. wilhelma were cultured in sterile ASW containing standard 24-well cell culture plates at $24^{\circ} \mathrm{C}$. On a daily basis, sponges were pulse-fed for 4.5 hours with freshly harvested induced, actin dsRNA-expressing HT115(DE3)/L4440-actb bacteria. A control group was pulse-fed with non-induced HT115 (DE3)/L4440-actb bacteria. Subsequently, in order to wash out the bacteria, the total volume was exchanged twice by aerated sterile ASW on a daily basis. Sponges were sampled for actin mRNA quantification 5 hours after bacteria wash-out every 24 hours for a period of 8 days (longterm experiment) and for a period of 2 days (short-term experiment). Samples were frozen in liquid nitrogen and stored at $-80^{\circ} \mathrm{C}$ until further batch processing. At the same time, samples were fixed for actin labeling and microscopy using $1.8 \%$ fomaldehyde and $0.2 \%$ glutaraldehyde in ASW at $4^{\circ} \mathrm{C}$ over night. After three washing steps (ASW), samples were dehydrated in an increasing Ethylalcohol (EtOH) series $(30 \%, 50 \%, 70 \%)$ and stored in $70 \% \mathrm{EtOH}$ until processed for microscopy.

\section{Time-lapse imaging}

Juvenile sponges of $T$. wilhelma in the long-term silencing experiment were time-lapse imaged for 22-24 hours prior to RNA extraction. One specimen of the RNAi treatment group and one specimen of the control group were imaged simultaneously. Images were taken every minute through the bottom of the 24-well plates. Standard web cameras (Logitech, QuickCam Pro for Notebooks) attached to a Laptop were used. Cameras were controlled by the video surveillance software Active WebCam Pro (Pysoft). Analysis of body contractions was performed in ImageJ [66] using custom made macros (available upon request) as previously described $[37,45]$.

\section{QRT-PCR validation of knock down}

E. muelleri sponges were collected after RNAi treatment and mRNA was isolated using the Qiagen RNeasy ${ }^{\circledR}$ Mini Kit and treated on columns with DNase I to limit contaminating genomic DNA. Equal amounts of cDNA (125-200 $\mathrm{ng} / \mu \mathrm{l}$ ) were synthesized from experimental and control sponge mRNA using Superscript III reverse transcriptase (Invitrogen) and oligodT primer. SYBR Green (Invitrogen) chemistry and the Chromo4 (BioRad) were used for qRTPCR using cycling conditions of $94^{\circ} \mathrm{C}$ for 3 min followed 
by $30 \mathrm{~s}$ at $94^{\circ} \mathrm{C}, 30 \mathrm{~s}$ at $55-61^{\circ} \mathrm{C}, 1 \mathrm{~min}$ at $72^{\circ} \mathrm{C}$ for 35 cycles with gene-specific primers (F: 5'-CGCTCTTCCCCACGCCATC-3'; R: 5'-TCGCTCGGCAGTGGTGGT-3'), which resulted in amplification of a $112 \mathrm{bp}$ fragment. Control experiments using Annexin utilized primer sequences F: 5'-TCTCGTTTTGCAATCTTGGCGTAT-3'; R: 5'-GG GTGCACGTGATGAGTCTCTT-3' and for Six1/2 F: 5'GCTTCAAGGAGAAGTCGCGTGT-3'; R: 5'-TGCCT CCGATTTTTGAACCAGT-3'. actin, Six1/2, and Annexin levels were normalized to Ef1a, a 'housekeeping' gene in E. muelleri that is consistently expressed at high levels throughout development. For all qRT-PCR experiments, duplicates were performed from master mixes. Threshold values for $\mathrm{Ct}$ calculation were selected by hand to optimize efficiency for all samples. Standard curves using plasmid dilutions as templates were made for each gene in each qPCR experiment and efficiency-corrected $\mathrm{Ct}$ values were compared to these curves to calculate relative concentrations using Opticon Monitor software. The relative concentration values of duplicates were averaged and experimental averages were normalized to Efla values.

T. wilhelma sponges were collected after RNAi treatment and homogenized with a potter. Total RNA was isolated by the RNA XS Kit (Macherey-Nagel) according to the manufacturer's instructions. cDNA was synthesized from experimental and control sponge mRNA with the First Strand cDNA Synthesis Kit (Fermentas) according to the manufacturer's instructions utilizing oligo $(\mathrm{dt})_{18}$ primers. SYBR Green (KAPA SYBR Fast qPCR Mastermix) chemistry and the Smart Cycler System (Cepheid/PeqLab) were used for qRT-PCR using cycling conditions of $94^{\circ} \mathrm{C}$ for 3 minutes followed by 45 cycles of $94^{\circ} \mathrm{C}$ for $30 \mathrm{~s}, 60^{\circ} \mathrm{C}$ for 30 seconds, $72^{\circ} \mathrm{C}$ for 45 seconds with gene specific primers Act-6_U_L (5'-GCTTGAAATCCTTCCCCTTC-3') and Act-6_U_R (5'-TCTACAACCAACGCAGCAAC-3') to amplify a 92-bp fragment of the $T$. wilhelma actin gene and TW12B02-RPS5-L (5'-ACTCCACTCGTATCGGT CGT-3') and TW12B02-RPS5-R (5'-AGAGGGGACACA TCAACAGC-3') to amplify a 61-bp fragment of the 40S ribosomal protein S5-related cluster (RPS5) as an internal reference for relative quantification of actin mRNA levels. For all qRT-PCR experiments, triplicates were performed from master mixes. We calculated amplification efficiencies for each replicon group and Cq values applying LinReg software (Version 12.5, http://LinRegPCR.nl) [67,68]. Efficiency-corrected relative quantification was conducted using the "efficiency-calibrated mathematical method for the relative expression ratio in real-time PCR" as used by the Roche LightCycler ${ }^{\circledR}$ software $[69,70]$.

\section{Western Blot validation of knock down}

Fifteen stage-five sponges were collected for each of the conditions: $d s E m-a c t b, d s E m$-six, and control-treated tissues. Lysis buffer (5\% $1 \mathrm{M}$ Tris pH 7.5, 6\% $5 \mathrm{M} \mathrm{NaCl}$, $0.4 \% 0.5$ M EDTA, $0.5 \%$ Triton-X, $0.2 \%$ protease inhibitor) was added to each of the samples. The sponges were mechanically ground using an RNAse-free pestle. Protein concentrations were calculated using a BioRad assay and $15 \mu \mathrm{g}$ of the protein were run on $12 \%$ Tris- $\mathrm{HCl}$ gels at $200 \mathrm{~V}$ at room temperature. Proteins were transferred to nitrocellulose membranes and were blocked overnight at $4^{\circ} \mathrm{C}$ to saturate unused protein binding sites. Blocking solution consisted of $5 \%$ nonfat dry milk in Tris Buffered Saline (TBS) buffer (pH of 7.4 and $0.01 \mathrm{M}$ Tris Base, $153 \mathrm{mM} \mathrm{NaCl}$ ). Membranes were washed in TBS and incubated overnight at $4^{\circ} \mathrm{C}$ with primary antibodies diluted to $100 \mu \mathrm{g} / \mu \mathrm{l}$ in TBST (TBS with 1\% Tween 20 and 2\% nonfat dry milk): Actin (h-300) rabbit polyclonal lgG or Six 1/2/4 (N-19) goat polyclonal lgG (Santa Cruz Biotechnology). The following day, nitrocellulose membranes were washed in TBST. Membranes were then incubated for 2 hours with gentle shaking at room temperature in corresponding secondary antibody: goat anti-rabbit lgG - 1:10000 FITC mouse/human absorbed or 1:10000 bovine anti-goat HRP (Santa Cruz Biotechnology). Membranes were washed in TBST and subsequently soaked in TBS for 5 minutes. Nitrocellulose membranes were detected with CDP star chemiluminescent substrate (Roche) for one minute, exposed to X-ray film and developed with an automatic film processor.

\section{Phalloidin staining and evaluation of actin phenotypes}

In E. muelleri, a protocol based on ref. [34] was utilized. E. muelleri gemmules were cultured and fed as above. At 22-26 hours post-feeding, sponges were fixed in 4\% paraformaldehyde in PBS at $4^{\circ} \mathrm{C}$ overnight. Fixed sponges were washed in PBS at room temperature for 10 minutes with gentle shaking in the dark. Autofluorescence was quenched by treating with $0.1 \mathrm{M}$ ammonium chloride for 5 minutes at room temperature. Tissue was permeabilized in $0.2 \%$ Triton in PBS for 10 minutes, followed by washing in cold PBS for 5 minutes. Coverslips with their attached sponges were inverted over Fastwell slides with rubber gaskets (Research Products International Corp.) containing $200 \mu \mathrm{L}$ of BODIPY 558/568 phalloidin (Invitrogen) at a concentration of $10 \mathrm{U} / \mathrm{mL}$. These were incubated at 3 hours in the dark at room temperature and then rinsed up to three times in PBS and mounted in fluoromount (Sigma). Fluorescence was visualized on a Leica SP2 LSCM scanning confocal microscope. Careful consideration was given to maintain settings across treatments.

In T. wilhelma, fixed and dehydrated samples were stepped through a decreasing series of $\mathrm{EtOH}$ in $\mathrm{PBS}$ (70\%, $50 \%, 30 \%)$. After three washing steps in PBS, samples were incubated in $0.1 \%$ Triton X-100 in PBS for 5 minutes, followed by three washing steps in PBS. Samples were preincubated for 30 minutes in BSA-PBS (PBS containing 1\% BSA) at $4{ }^{\circ} \mathrm{C}$ followed by Alexa Fluor 488 phalloidin 
staining in BSA-PBS. After washing for three times in PBS, samples were mounted in ProLong Gold antifading solution. Confocal imaging was performed on a Zeiss 510 cLSM, using an Argon laser for excitation at $488 \mathrm{~nm}$ and a $515 \mathrm{~nm}$ long pass emission filter at equal settings for RNAi-treated and control specimens.

\section{Additional material}

Additional file 1: Supplementary Figures. Figure S1. Ephydatia muelleri and Tethya wilhelma actin sequences. Figure S2. Ephydatia muelleri and Tethya wilhelma dsRNA expression vector. Figure S3. Maximum likelihood (RAXML) actin gene family analysis including Ephydatia muelleri and Tethya wilhelma actin genes used for RNAi knockdown. Figure S4. Nucleotide alignment of Tethya wilhelma and Ephydatia muelleri partial actin sequences in comparison to actin of other organisms. Figure S5. Translated amino acid alignment of Tethya wilhelma and Ephydatia muelleri partial actin sequences in comparison to actin of other organisms. Figure S6. Actin dsRNA treatment by soaking method and recovery of sponge tissue after removal of actin dsRNA. Figure S7. RTPCR and gRT-PCR analysis of dsEm-Annexin and dsEm-Six treatments. Figure S8. Western blot analysis of RNAi- and control-treated tissues. Figure S9. Viability check of RNAi-treated specimens of T. wilhelma. Figure S10. Cross-section of choanocyte chambers from treatment and control sponges. Figure S11. Exopinacocyte morphology of T. wilhelma and possible fixation artifacts demonstrated by scanning electron microscopy.

\section{Additional File 2: Viability check of RNAi-treated T. wilhelma} specimens. Time-lapse imaging and image analysis was used to address contractility as a viability marker. Time-lapse image movie of the specimen representing the knockdown value at 201 hours in the longterm study (Figure $3 \mathrm{C}$ and Figure 4). The specimen is dislocated at the time of RNAi feeding due to liquid handling (time: 12:24 hh:mm; see Figure 4; culture dish had to be temporarily removed from the imaging station for feeding and was moved back afterwards).

Additional File 3: Nucleotide sequence alignment of contig assemblies of cytoplasmic actin complete CDS from selected Holozoa/Metazoa (fasta format).

Additional File 4: Translated amino acid sequence alignment of the complete actin CDS shown in Additional File 3(fasta format).

\section{Acknowledgements}

We are grateful to Martin S. Fischer (Jena) for supporting our work and providing infrastructure, Erika Kothe (Jena), Michael Friedrich and Peqlab Biotechnologie $\mathrm{GmbH}$ (Erlangen) for providing access to qPCR machines, Jürgen Bolz, Michael Richter \& André Steinecke (Jena) for access to and assistance at the CLSM, Isabel Koch and Alex Mendosa (both Stuttgart) for access to sponge material supplementing our own culture, and Mareike Klinger, Katja Felbel and Benjamin Weiss (all Jena) for excellent technical support. JUH received funding from Studienstiftung des Deutschen Volkes. MN was supported through FSU Jena DRM program. GW acknowledges funding from the German Science Foundation (DFG). We are also grateful to Carolyn Marks for help with confocal and electron microscopy (Richmond). We thank Heather Babka (ISU) and Martin Dohrmann (Smithsonian Institution) for comments on a previous version of the manuscript. ALH was supported by a grant from the Thomas F. and Kate Miller Jeffress Memorial Trust and ALH, MSH and DVL acknowledge funding from the National Science Foundation (AToL).

\section{Author details}

'Department of Biology, University of Richmond, Richmond, VA, USA. ${ }^{2}$ Institut für Spezielle Zoologie und Evolutionsbiologie mit Phyletischem Museum, Friedrich-Schiller-Universität, Jena, Germany. ${ }^{3}$ lowa State University, Ames, IA, USA. ${ }^{4}$ Department of Earth and Environmental Sciences \& GeoBioCenterLMU, Ludwig-Maximilians-University Munich, Germany. ${ }^{5}$ Biology Department, College of the Pacific, Stockton, CA, USA. ${ }^{6}$ Molecular Cell
Biology, Washington University, St. Louis, MO, USA. ${ }^{7}$ Molecular and Cellular Biology, Stony Brook University, Stony Brook, NY, USA. ${ }^{8}$ Sigma-Aldrich Biotechnology, St. Louis, MO, USA.

\section{Authors' contributions}

E. muelleri experiments: AR adapted the feeding method, carried out molecular analyses, performed confocal microscopy analysis of phalloidin staining and co-drafted the manuscript. ED participated in the design of the study, developed the dsRNA soaking method and performed all control experiments for soaking methodology. BC made the dsRNA constructs for the feeding method, piloted feeding protocols and developed standards for qPCR. IW conducted molecular analyses and worked on optimization of feeding methods, as well as confocal microscopy analysis of phalloidin staining. DP conducted Western blot analysis for Actin and Six proteins, assayed annexin expression after RNAi, and worked extensively on manuscript revisions. SK participated in the design of both the soaking and feeding methods (including appropriate controls), provided vectors, protocols, and GFP constructs, and aided with data interpretation. MH collected Ephydatia tissue, conducted TEM analysis, evaluated statistical methods, and revised the manuscript. AH conceived of the study, obtained funding for the study, participated in the design and implementation of all experiments and co-drafted the manuscript. T. wilhelma experiments: $\mathrm{JH}$ adapted the feeding method, carried out molecular analyses, performed confocal microscopy analysis of phalloidin staining, prepared figure drafts and co-drafted the manuscript. KMH carried out molecular analyses and constructed the feeding vector. DVL conceived parts of the study, provided CDNA data and co-drafted the manuscript. GW provided EST data, performed the gene analysis and contributed to the manuscript. MN conceived of the study, obtained funding for the study, participated in design and implementation, performed confocal microscopy analysis, prepared final figures and co-drafted the manuscript. All authors have read and approved the final manuscript.

Received: 9 December 2010 Accepted: 16 June 2011 Published: 16 June 2011

\section{References}

1. Dunn CW, Hejnol A, Matus DQ, Pang K, Browne WE, Smith SA, Seaver E, Rouse GW, Obst M, Edgecombe GD, et al: Broad phylogenomic sampling improves resolution of the animal tree of life. Nature 2008, 452:745-749.

2. Philippe $H$, Derelle R, Lopez P, Pick K, Borchiellini C, Boury-Esnault N, Vacelet J, Renard E, Houiliston E, Quéinnec E, et al: Phylogenomics Revives Traditional Views on Deep Animal Relationships. Current Biology 2009, 19:1-7.

3. Pick KS, Philippe H, Schreiber F, Erpenbeck D, Jackson DJ, Wrede P, Wiens M, Alié A, Morgenstern B, Manuel M, Wörheide G: Improved Phylogenomic Taxon Sampling Noticeably Affects Nonbilaterian Relationships. Molecular Biology and Evolution 2010, 27:1983-1987.

4. Schierwater B, Eitel M, Jakob W, Osigus HJ, Hadrys H, Dellaporta SL, Kolokotronis SO, DeSalle R: Concatenated Analysis Sheds Light on Early Metazoan Evolution and Fuels a Modern "Urmetazoon" Hypothesis. PLoS Biology 2009, 7(1):e1000020 [http://www.plosbiology.org/article/info\%3Adoi \%2F10.1371\%2Fjournal.pbio.1000020].

5. Sperling EA, Peterson KJ, Pisani D: Phylogenetic-Signal Dissection of Nuclear Housekeeping Genes Supports the Paraphyly of Sponges and the Monophyly of Eumetazoa. Molecular Biology and Evolution 2009, 26:2261-2274.

6. King N, Westbrook MJ, Young SL, Kuo A, Abedin M, Chapman J. Fairclough $\mathrm{S}$, Hellsten $U$, Isogai $Y$, Letunic $I$, et al: The genome of the choanoflagellate Monosiga brevicollis and the origin of metazoans. Nature 2008, 451:783-788.

7. Srivastava M, Begovic E, Chapman J, Putnam NH, Hellsten U, Kawashima T, Kuo A, Mitros T, Salamov A, Carpenter ML, et al: The Trichoplax genome and the nature of placozoans. Nature 2008, 454:955-960.

8. Srivastava M, Simakov O, Chapman J, Fahey B, Gauthier MEA, Mitros T, Richards GS, Conaco C, Dacre M, Hellsten U, et al: The Amphimedon queenslandica genome and the evolution of animal complexity. Nature 2010, 466:720-726.

9. Adamska M, Larroux C, Adamski M, Green K, Lovas E, Koop D, Richards GS, Zwafink C, Degnan BM: Structure and expression of conserved Wnt 
pathway components in the demosponge Amphimedon queenslandica. Evolution \& Development 2010, 12:494-518.

10. Adamska M, Matus DQ, Adamski M, Green K, Rokhsar DS, Martindale MQ, Degnan BM: The evolutionary origin of hedgehog proteins. Current Biology 2007, 17:R836-R837.

11. Gauthier MEA, Du Pasquier L, Degnan BM: The genome of the sponge Amphimedon queenslandica provides new perspectives into the origin of Toll-like and interleukin 1 receptor pathways. Evolution \& Development 2010, 12:519-533.

12. Kosik KS: Exploring the early origins of the synapse by comparative genomics. Biology Letters 2009, 5:108-111.

13. Nickel M: Evolutionary emergence of synaptic nervous systems: what can we learn from the non-synaptic, nerveless Porifera? Invertebrate Biology 2010, 129:1-16.

14. Richards GS, Simionato E, Perron M, Adamska M, Vervoort M, Degnan BM: Sponge Genes Provide New Insight into the Evolutionary Origin of the Neurogenic Circuit. Current Biology 2008, 18:1156-1161.

15. Sakarya O, Armstrong KA, Adamska M, Adamski M, Wang IF, Tidor B, Degnan BM, Oakley TH, Kosik KS: A Post-Synaptic Scaffold at the Origin of the Animal Kingdom. PLOS ONE 2007, 2:e506.

16. Jackson DJ, Macis L, Reitner J, Degnan BM, Worheide G: Sponge paleogenomics reveals an ancient role for carbonic anhydrase in skeletogenesis. Science 2007, 316:1893-1895.

17. Lavrov DV, Wang $X$, Kelly M: Reconstructing ordinal relationships in the Demospongiae using mitochondrial genomic data. Molecular Phylogenetics and Evolution 2008, 49:111-124

18. Jakob W, Sagasser S, Dellaporta S, Holland P, Kuhn K, Schierwater B: The Trox-2 Hox/ParaHox gene of Trichoplax (Placozoa) marks an epithelial boundary. Development Genes and Evolution 2004, 214:170-175.

19. Jakob W, Schierwater B: Changing Hydrozoan Bauplans by Silencing HoxLike Genes. PLOS ONE 2007, 2:e694.

20. Lohmann JU, Endl I, Bosch TCG: Silencing of Developmental Genes in Hydra. Developmental Biology 1999, 214:211-214.

21. Pankow S, Bamberger C: The p53 Tumor Suppressor-Like Protein nvp63 Mediates Selective Germ Cell Death in the Sea Anemone Nematostella vectensis. PLOS ONE 2007, 2:e782.

22. Yamada A, Martindale MQ, Fukui A, Tochinai S: Highly conserved functions of the Brachyury gene on morphogenetic movements: Insight from the early-diverging phylum Ctenophora. Developmental Biology 2010, 339:212-222.

23. Gee L, Hartig J, Law L, Wittlieb J, Khalturin K, Bosch TCG, Bode HR: [beta]catenin plays a central role in setting up the head organizer in hydra. Developmental Biology 2010, 340:116-124.

24. Timmons $L$, Court DL, Fire A: Ingestion of bacterially expressed dsRNAs can produce specific and potent genetic interference in Caenorhabditis elegans. Gene 2001, 263:103-112.

25. Timmons L, Fire A: Specific interference by ingested dsRNA. Nature 1998 , 395:854-854.

26. Fire A, Xu S, Montgomery MK, Kostas SA, Driver SE, Mello CC: Potent and specific genetic interference by double-stranded RNA in Caenorhabditis elegans. Nature 1998, 391:806-811.

27. Dunn SR, Phillips WS, Green DR, Weis VM: Knockdown of Actin and Caspase Gene Expression by RNA Interference in the Symbiotic Anemone Aiptasia pallida. Biological Bulletin 2007, 212:250-258.

28. Lee H, Kimelman D: A Dominant-Negative Form of p63 is Required for Epidermal Proliferation in Zebrafish. Developmental Cell 2002, 2:607-616.

29. Lekven AC, Thorpe CJ, Waxman JS, Moon RT: Zebrafish wnt8 Encodes Two Wnt8 Proteins on a Bicistronic Transcript and Is Required for Mesoderm and Neurectoderm Patterning. Developmental Cell 2001, 1:103-114.

30. Chera S, de Rosa R, Miljkovic-Licina M, Dobretz K, Ghila L, Kaloulis K, Galliot B: Silencing of the hydra serine protease inhibitor Kazal1 gene mimics the human SPINK1 pancreatic phenotype. J Cell Sci 2006, 119:846-857.

31. Manconi R, Pronzato R: Suborder Spongillina subord. nov.: Freshwater Sponges. In Systema Porifera: A Guide to the Classification of Sponges. Volume 1. Edited by: Hooper JNA, Van Soest RWM. New York: Kluwer Academic/Plenum Publishers New York; 2002:921-1001.

32. Funayama $\mathrm{N}$ : The stem cell system in demosponges: Insights into the origin of somatic stem cells. Development, Growth \& Differentiation 2010, $52: 1-14$
33. Adams ED, Goss GG, Leys SP: Freshwater sponges have functional, sealing epithelia with high transepithelial resistance and negative transepithelial potential. PLoS One 2010, 5:e15040.

34. Elliott GRD, Leys SP: Coordinated contractions effectively expel water from the aquiferous system of a freshwater sponge. J Exp Biol 2007, 210:3736-3748.

35. Windsor PJ, Leys SP: Wnt signaling and induction in the sponge aquiferous system: evidence for an ancient origin of the organizer. Evol Dev 2010, 12:484-493.

36. Funayama N, Nakatsukasa M, Hayashi T, Agata K: Isolation of the choanocyte in the fresh water sponge, Ephydatia fluviatilis and its lineage marker, Ef annexin. Development, Growth \& Differentiation 2005, 47:243-253.

37. Nickel M: Kinetics and rhythm of body contractions in the sponge Tethya wilhelma (Porifera: Demospongiae). Journal of Experimental Biology 2004, 207:4515-4524.

38. Sarà M, Sarà A, Nickel M, Brümmer F: Three new species of Tethya (Porifera: Demospongiae) from German aquaria. Stuttgarter Beiträge zur Naturkunde Serie A 2001, 631:1-15.

39. Hammel JU, Herzen J, Beckmann F, Nickel M: Sponge budding is a spatiotemporal morphological patterning process: Insights from synchrotron radiation-based $\mathrm{x}$-ray microtomography into the asexual reproduction of Tethya wilhelma. Frontiers in Zoology 2009, 6:19.

40. Hammel JU, Filativ MV, Herzen J, Beckmann F, Kaandorp J, Nickel M: Acta Zoologica 2011, 92: [http://onlinelibrary.wiley.com/doi/10.1111/j.14636395.2010.00492.x/full].

41. Nickel M, Donath T, Schweikert M, Beckmann F: Functional morphology of Tethya species (Porifera): 1. quantitative 3D-analysis of $T$. wilhelma by synchrotron radiation based $\mathrm{x}$-ray microtomography. Zoomorphology 2006, 125(4):209-223

42. Nickel M, Scheer C, Hammel JU, Herzen J, Beckmann F: The contractile sponge epithelium sensu lato - body contraction of the demosponge Tethya wilhelma is mediated by the pinacoderm. The Journal of Experimental Biology 2011, 214:1692-1698.

43. Nickel M: Like a 'rolling stone': quantitative analysis of the body movement and skeletal dynamics of the sponge Tethya wilhelma. Journal of Experimental Biology 2006, 209:2839-2846.

44. Ellwanger K, Eich A, Nickel M: GABA and Glutamate specifically induce contractions in the sponge Tethya wilhelma. Journal of Comparative Physiology A, Sensory, Neural, and Behavioral Physiology 2007, 193(1):1-11.

45. Ellwanger $\mathrm{K}$, Nickel $\mathrm{M}$ : Neuroactive substances specifically modulate rhythmic body contractions in the nerveless metazoon Tethya wilhelma (Demospongiae, Porifera). Frontiers in Zoology 2006, 3:7.

46. Nakajima-lijima S, Hamada H, Reddy P, Kakunaga T: Molecular structure of the human cytoplasmic beta-actin gene: interspecies homology of sequences in the introns. Proceedings of the National Academy of Sciences of the United States of America 1985, 82:6133-6137.

47. Bairoch A, Bucher P, Hofmann K: The PROSITE database, its status in 1997. Nucleic Acids Research 1997, 25:217-221.

48. Altschul SF, Madden TL, Schäffer AA, Zhang J, Zhang Z, Miller W, Lipman DJ: Gapped BLAST and PSI-BLAST: a new generation of protein database search programs. Nucleic Acids Research 1997, 25:3389-3402

49. Sehring I, Mansfeld J, Reiner C, Wagner E, Plattner H, Kissmehl R: The actin multigene family of Paramecium tetraurelia. BMC Genomics 2007, 8:82.

50. Boguski MS, Lowe TMJ, Tolstoshev CM: dbEST [mdash] database for [Idquo]expressed sequence tags[rdquo]. Nat Genet 1993, 4:332-333.

51. Hill M, Stabile C, Steffen LK, Hill A: Toxic effects of endocrine disrupters on freshwater sponges: common developmental abnormalities. Environmental Pollution 2002, 117:295-300.

52. Nickel M, Brümmer F: Body extension types of Tethya wilhelma: cellular organisation and their function in movement. Bollettino dei musei e degli istituti biologici dell' Universita di Genova 2004, 68:483-489.

53. Kabsch W, Vandekerckhove J: Structure and Function of Actin. Annual Review of Biophysics and Biomolecular Structure 2003, 21:49-76.

54. De Goeij JM, De Kluiijver A, Van Duyl FC, Vacelet J, Wijffels RH, De Goeij AFPM, Cleutjens JPM, Schutte B: Cell kinetics of the marine sponge Halisarca caerulea reveal rapid cell turnover and shedding. Journal of Experimental Biology 2009, 212:3892-3900.

55. Arendt $D$ : The evolution of cell types in animals: emerging principles from molecular studies. Nature Reviews Genetics 2008, 9:868-882. 
56. Ereskovsky A, Tokina D: Asexual reproduction in homoscleromorph sponges (Porifera; Homoscleromorpha). Marine Biology 2007, 151:425-434.

57. Ereskovsky AV, Borchiellini C, Gazave E, Ivanisevic J, Lapébie P, Perez T, Renard E, Vacelet J: The Homoscleromorph sponge Oscarella lobularis, a promising sponge model in evolutionary and developmental biology. BioEssays 2009, 31:89-97.

58. Manuel M, Le Parco Y: Homeobox Gene Diversification in the Calcareous Sponge, Sycon raphanus. Molecular Phylogenetics and Evolution 2000, 17:97-107.

59. Manuel M, Borchiellini C, Alivon E, Le PY, Vacelet J, Boury-Esnault N: Phylogeny and evolution of calcareous sponges: Monophyly of Calcinea and Calcaronea, high level of morphological homoplasy, and the primitive nature of axial symmetry. Systematic Biology 2003, 52:311-333.

60. Adamska M, Degnan B, Green K, Zwafink C: What sponges can tell us about the evolution of developmental processes. Zoology 2011, 114:1-10.

61. Agata K, Nakajima E, Funayama N, Shibata N, Saito Y, Umesono Y: Two different evolutionary origins of stem cell systems and their molecular basis. Seminars in Cell \& Developmental Biology 2006, 17:503-509.

62. Funayama N, Nakatsukasa M, Kuraku S, Takechi K, Dohi M, Iwabe N, Miyata T, Agata K: Isolation of Ef silicatein and Ef lectin as Molecular Markers Sclerocytes and Cells Involved in Innate Immunity in the Freshwater Sponge Ephydatia fluviatilis. Zoological Science 2005, 22:1113-1122

63. Funayama N, Nakatsukasa M, Mohri K, Masuda Y, Agata K: Piwi expression in archeocytes and choanocytes in demosponges: insights into the stem cell system in demosponges. Evolution \& Development 2010, 12:275-287.

64. Strekal TA, McDiffett WF: Factors affecting germination, growth, and distribution of the freshwater sponge, Spongilla fragilis Leidy (Porifera). Biol Bull 1974, 146:267-278.

65. Stamatakis A: RAxML-VI-HPC: maximum likelihood-based phylogenetic analyses with thousands of taxa and mixed models. Bioinformatics 2006, 22:2688-2690.

66. Rasband WS: ImageJ Bethesda, Maryland, USA: U. S. National Institutes of Health; 1997 [http://rsb.info.nih.gov/ij]].

67. Ramakers C, Ruijter JM, Deprez RHL, Moorman AFM: Assumption-free analysis of quantitative real-time polymerase chain reaction (PCR) data. Neuroscience Letters 2003, 339:62-66.

68. Ruijter JM, Ramakers C, Hoogaars WMH, Karlen Y, Bakker O, van den Hoff MJB, Moorman AFM: Amplification efficiency: linking baseline and bias in the analysis of quantitative PCR data. Nucleic Acids Research 2009, 37:e45-e45.

69. Pfaffl MW: A new mathematical model for relative quantification in realtime RT-PCR. Nucleic Acids Research 2001, 29:e45-e45.

70. Soong R, Ruschoff J, Tabiti K: Detection of colorectal micrometastasis by quantitative RT-PCR of cytokeratin 20 mRNA. Roche Molecular Biochemicals Internal Publication 2000.

doi:10.1186/1472-6750-11-67

Cite this article as: Rivera et al:: RNA interference in marine and freshwater sponges: actin knockdown in Tethya wilhelma and Ephydatia muelleri by ingested dsRNA expressing bacteria. BMC Biotechnology 2011 11:67.

\section{Submit your next manuscript to BioMed Central and take full advantage of:}

- Convenient online submission

- Thorough peer review

- No space constraints or color figure charges

- Immediate publication on acceptance

- Inclusion in PubMed, CAS, Scopus and Google Scholar

- Research which is freely available for redistribution

Submit your manuscript at www.biomedcentral.com/submit
Biomed Central 